\title{
The Fundamental Matrix of the System of Linear Micropolar Elasticity
}

\author{
Jerzy Gawinecki, Gerhard Kirchner, Jarosław Lazuka
}

\begin{abstract}
We construct the fundamental matrix of the system of partial differential operators governing the motion of linear micropolar elastic media in terms of derivatives of distributions which are convolutions of the fundamental solutions to the wave operator, the Klein-Gordon operator and the micropolar operator.
\end{abstract}

Keywords: Fundamental solutions, linear partial differential operators with constant coefficients, evolution operators, asymmetric elasticity

MSC 2000: 35E05, 35C05, 74A60, 74B99

\section{Introduction and notations}

The theory of an elastic continuum with oriented particles, the so-called micropolar medium or Cosserat medium, was created by two French scholars, the brothers E. and F. Cosserat in 1909 (cf. [1]).

The foundations of this theory lay in a discrepancy between the classical theory of elasticity and certain experiments, which becomes particularly obvious in dynamical problems, namely in the case of ultrasonic waves, vibrations of granular bodies and bodies with large molecules, such as polymers. In micropolar bodies the influence of the microstructure must be taken into consideration and with each material particle is associated a perfectly rigid triad which in the course of deformation can undergo not only a displacement but also a rotation. As particles in an asymmetric elastic medium dispose of an orientation, the deformation of the body is described by the displacement vector $\mathbf{u}(t, \mathbf{x})$ and an independent rotation vector $\boldsymbol{\varphi}(t, \mathbf{x})$. The purpose of this paper is the construction of the fundamental matrix of the system of partial differential equations

J. Gawinecki: Institute of Mathematics and Cryptology, Military University of Technology, Kaliskiego Str. 2, 00-908 Warsaw, Poland; j.gawinecki@imbo.wat.edu.pl

G. Kirchner: Institut für Mathematik,Universität Innsbruck, Technikerstraße 25, 6020 Innsbruck, Austria; Gerhard.Kirchner@uibk.ac.at

J. Łazuka: Institute of Mathematics and Cryptology, Military University of Technology, Kaliskiego Str. 2, 00-908 Warsaw, Poland; j.lazuka@imbo.wat.edu.pl

ISSN 0232-2064 / \$ 2.50 (c) Heldermann Verlag Berlin 
describing the motion of linear, homogeneous, isotropic and centrally symmetric micropolar elastic bodies, which system has been derived by W. Nowacki and is presented in [5] (cf. also[2]).

We use the following terminology and notations: By $I_{3}$ we denote the $3 \times 3$ identity matrix and the letters $\mathbf{u}, \boldsymbol{\varphi}$ are used for vector fields on $\mathbb{R}^{3}$, the Heaviside function is denoted by $Y . S^{2}$ denotes the unit sphere in $\mathbb{R}^{3}$ and $\mathrm{d} \omega$ the surface measure on $S^{2}$, whereas $B_{2}$ is the unit disc in $\mathbb{R}^{2} . \mathcal{S}^{\prime}$ is the space of tempered distributions. We use the following differentiation symbols:

$$
\partial_{t}=\frac{\partial}{\partial t}, \quad \partial_{i}=\frac{\partial}{\partial x_{i}}, \quad \nabla=\left(\partial_{1}, \partial_{2}, \partial_{3}\right)^{\mathrm{T}}, \quad \partial=\left(\partial_{t}, \nabla^{\mathrm{T}}\right), \quad \Delta_{3}=\partial_{1}^{2}+\partial_{2}^{2}+\partial_{3}^{2}
$$

and the following abbreviations for some frequently used scalar differential operators:

$$
\begin{aligned}
W(\partial) & =\rho \partial_{t}^{2}-(\mu+\alpha+a) \Delta_{3} \\
K(\partial) & =J \partial_{t}^{2}-(\gamma+\varepsilon+b) \Delta_{3}+4 \alpha \\
\tilde{W}(\partial) & =\rho \partial_{t}^{2}-(\mu+\alpha) \Delta_{3} \\
\tilde{K}(\partial) & =J \partial_{t}^{2}-(\gamma+\varepsilon) \Delta_{3}+4 \alpha \\
T(\partial) & =\tilde{W}(\partial) \tilde{K}(\partial)+4 \alpha^{2} \Delta_{3} \\
& =\left(\rho \partial_{t}^{2}-(\mu+\alpha) \Delta_{3}\right)\left(J \partial_{t}^{2}-(\gamma+\varepsilon) \Delta_{3}\right)+4 \alpha \rho \partial_{t}^{2}-4 \alpha \mu \Delta_{3} \\
T_{\operatorname{pr}}(\partial) & =\left(\rho \partial_{t}^{2}-(\mu+\alpha) \Delta_{3}\right)\left(J \partial_{t}^{2}-(\gamma+\varepsilon) \Delta_{3}\right) .
\end{aligned}
$$

For an operator $P(\partial)$ which is hyperbolic in direction $N=(1,0,0,0)$, we denote by $E_{P}$ its unique fundamental solution with support in the half-space $t \geq 0$, cf. [4], Theorem 12.5.1.

\section{The system of differential equations for a micropolar elastic body}

The influence of external loads $X$ and $\Phi$ on a body results in a displacement $\mathbf{u}(t, \mathbf{x})$ and a rotation $\boldsymbol{\varphi}(t, \mathbf{x})$. The relations fulfilled by these functions at a space point $\mathbf{x} \in \mathbb{R}^{3}$ at the time $t$ for a linear, homogeneous, isotropic and centrally symmetric micropolar elastic body have the form (cf. [5, p. 22, Section 1.7., Equ. (6)f.]

$$
\begin{aligned}
& \rho \ddot{\mathbf{u}}=(\mu+\alpha) \Delta_{3} \mathbf{u}+(\lambda+\mu-\alpha) \nabla \nabla^{\mathrm{T}} \mathbf{u}+2 \alpha \operatorname{curl} \boldsymbol{\varphi}+X \\
& J \ddot{\boldsymbol{\varphi}}=(\gamma+\varepsilon) \Delta_{3} \boldsymbol{\varphi}+(\beta+\gamma-\varepsilon) \nabla \nabla^{\mathrm{T}} \boldsymbol{\varphi}-4 \alpha \boldsymbol{\varphi}+2 \alpha \operatorname{curl} \mathbf{u}+\Phi,
\end{aligned}
$$

where $\mathbf{u}$ is the displacement vector, $\boldsymbol{\varphi}$ the rotation vector and $\mu, \lambda, \alpha, \beta, \varepsilon, \gamma, \rho, J$ are real constants satisfying the conditions $\mu>0,3 \lambda+2 \mu>0, \gamma>0,3 \beta+2 \gamma>$ 
$0, \alpha>0, \varepsilon>0$. The six equations in (1) and (2) can be written in matrix form as

$$
P(\partial)\left(\begin{array}{l}
\mathbf{u} \\
\varphi
\end{array}\right)=\left(\begin{array}{l}
X \\
\Phi
\end{array}\right)
$$

where $P(\partial)$, the matrix of partial differential operators of asymmetric elasticity, has the form

$$
P(\partial)=\left(\begin{array}{cc}
\tilde{W}(\partial) I_{3}-a \nabla \nabla^{\mathrm{T}} & A(\nabla) \\
A(\nabla) & \tilde{K}(\partial) I_{3}-b \nabla \nabla^{\mathrm{T}}
\end{array}\right)
$$

where

$$
A(\nabla)=2 \alpha\left(\begin{array}{ccc}
0 & \partial_{3} & -\partial_{2} \\
-\partial_{3} & 0 & \partial_{1} \\
\partial_{2} & -\partial_{1} & 0
\end{array}\right)
$$

and $a=\lambda+\mu-\alpha, b=\beta+\gamma-\varepsilon$.

\section{The fundamental matrix of the system of linear micropolar elasticity}

The construction of a fundamental matrix $E_{P}$ of the system (3) can be reduced to the construction of a fundamental solution $E_{D}$ of the scalar operator $D(\partial)=$ $\operatorname{det} P(\partial)$ by the formula $E_{P}=P^{\text {ad }}(\partial) E_{D}$. Therein $P^{\text {ad }}(\partial)$ denotes the adjoint matrix of $P(\partial)$, namely

$$
P^{\mathrm{ad}}(\partial)=\left(\begin{array}{ll}
P_{1}^{\mathrm{ad}}(\partial) & P_{2}^{\mathrm{ad}}(\partial) \\
P_{2}^{\mathrm{ad}}(\partial) & P_{3}^{\mathrm{ad}}(\partial)
\end{array}\right)
$$

where

$$
\begin{aligned}
& P_{1}^{\mathrm{ad}}(\partial)=K(\partial) T(\partial)\left(\tilde{K}(\partial) W(\partial) I_{3}+\left(a \tilde{K}(\partial)+4 \alpha^{2}\right) \nabla \nabla^{\mathrm{T}}\right) \\
& P_{2}^{\mathrm{ad}}(\partial)=-W(\partial) K(\partial) T(\partial) A(\nabla) \\
& P_{3}^{\mathrm{ad}}(\partial)=W(\partial) T(\partial)\left(\tilde{W}(\partial) K(\partial) I_{3}+\left(b \tilde{W}(\partial)+4 \alpha^{2}\right) \nabla \nabla^{\mathrm{T}}\right) .
\end{aligned}
$$

The matrix $P(t, \mathbf{x})$ has the eigenvectors $\left(\begin{array}{l}\mathbf{x} \\ 0\end{array}\right)$ and $\left(\begin{array}{l}0 \\ \mathbf{x}\end{array}\right)$ with the respective eigenvalues $W(t, \mathbf{x})$ and $K(t, \mathbf{x})$ and this leads to the following factorization of the determinant $D(\partial)=\operatorname{det} P(\partial)$ :

$$
D(\partial)=W(\partial) K(\partial) T^{2}(\partial),
$$

i. e. $P(\partial)$ is the product of the wave operator $W(\partial)$, the Klein-Gordon-operator $K(\partial)$ and the square of the operator $T(\partial)$. In the following, $T(\partial)$ will be referred 
to as the micropolar operator. In Section 4 it will be shown that the operator $T(\partial)$ is hyperbolic with respect to $N=(1,0,0,0)$. As a product of operators which are hyperbolic in $t$-direction, the operator $D(\partial)=W(\partial) K(\partial) T^{2}(\partial)$ is also hyperbolic in $t$-direction and therefore possesses a unique fundamental solution $E_{D}$ with support in the half-space $t \geq 0$, which shows that the fundamental matrix $E_{P}$ of the operator $P(\partial)$ is unique and the supports of its entries are contained in the half-space $t \geq 0$. The fundamental solution of $D(\partial)$ is given by $E_{D}=E_{W} * E_{K} * E_{T^{2}}$. Upon applying the matrix operator $P^{\text {ad }}(\partial)$ given in (4) to $E_{D}$ some factors cancel resulting in the following formula for the fundamental matrix $E_{P}$ of $P(\partial)$ :

$$
E_{P}=\left(\begin{array}{cc}
\tilde{K}(\partial) E_{T} I_{3}+M(\partial) E_{W} * E_{T} & -A(\nabla) E_{T} \\
-A(\nabla) E_{T} & \tilde{W}(\partial) E_{T} I_{3}+N(\partial) E_{K} * E_{T}
\end{array}\right)
$$

with

$$
\begin{aligned}
M(\partial) & :=\left(a \tilde{K}(\partial)+4 \alpha^{2}\right) \nabla \nabla^{\mathrm{T}} \\
N(\partial) & :=\left(b \tilde{W}(\partial)+4 \alpha^{2}\right) \nabla \nabla^{\mathrm{T}}
\end{aligned}
$$

\section{The fundamental solution of the micropolar operator}

For the construction of the fundamental solution of the micropolar operator we use the following theorem ([7, Proposition 2, p. 447].

Theorem 1. Let $N \in \mathbb{R}^{n} \backslash\{0\}$ and

$$
P_{\lambda}(\partial)=P_{0}(\partial)+\lambda_{1} P_{1}(\partial)+\lambda_{2} P_{2}(\partial)+\lambda_{3} P_{3}(\partial)
$$

where $\lambda=\left(\lambda_{1}, \lambda_{2}, \lambda_{3}\right) \in S^{2}$, be a family of uniformly quasihyperbolic operators with respect to $N$, i. e.

$$
\exists \sigma_{0} \in \mathbb{R}: \forall \sigma>\sigma_{0}: \forall \xi \in \mathbb{R}^{n}: \forall \lambda \in S^{2}: P_{\lambda}(\sigma N+i \xi) \neq 0 .
$$

If $E_{\lambda}$ denotes the uniquely determined fundamental solution to $P_{\lambda}(\partial)^{2}$ with the properties

(i) $\operatorname{supp} E_{\lambda} \subseteq\left\{\mathbf{x} \in \mathbb{R}^{n} ;\langle\mathbf{x}, N\rangle \geq 0\right\}$

(ii) $\mathrm{e}^{-\sigma\langle\mathbf{x}, N\rangle} E_{\lambda} \in \mathcal{S}^{\prime}$ for some $\sigma$ as in (5),

then the operator $P(\partial):=P_{0}(\partial)^{2}-P_{1}(\partial)^{2}-P_{2}(\partial)^{2}-P_{3}(\partial)^{2}$ is also quasihyperbolic with respect to $N$ and its fundamental solution $E$ with the properties (6) and (7) is given by

$$
E=\frac{1}{4 \pi} \int_{S^{2}} E_{\lambda} \mathrm{d} \omega(\lambda)
$$


Remark. We will apply Theorem 1 in the case $P(\partial)=P_{0}(\partial)^{2}-P_{1}(\partial)^{2}-P_{2}(\partial)^{2}$, i. e. $P_{3}(\partial)=0$. This yields the formula (cf. [7, Remark in p. 448])

$$
E=\frac{1}{2 \pi} \int_{B_{2}} E_{\lambda} \frac{\mathrm{d} \lambda}{\sqrt{1-|\lambda|^{2}}}
$$

The micropolar operator $T(\partial)$ can be written in the form $T(\partial)=\rho J \tilde{T}$, where

$$
\tilde{T}(\partial)=\partial_{t}^{4}-2 c \Delta_{3} \partial_{t}^{2}+2 d \partial_{t}^{2}+\left(c^{2}-e^{2}\right) \Delta_{3}^{2}-2(c d-e f) \Delta_{3}
$$

with

$$
\begin{aligned}
& c:=\frac{1}{2}\left(\frac{\mu+\alpha}{\rho}+\frac{\gamma+\varepsilon}{J}\right), d:=\frac{2 \alpha}{J}, e:=\frac{1}{2}\left|\frac{\mu+\alpha}{\rho}-\frac{\gamma+\varepsilon}{J}\right| \\
& f:=\frac{1}{e}\left(c d-\frac{2 \alpha \mu}{\rho J}\right)=\frac{\alpha}{e J}\left(\frac{\alpha-\mu}{\rho}+\frac{\gamma+\varepsilon}{J}\right) .
\end{aligned}
$$

For $e \neq 0$ we can now represent $\tilde{T}(\partial)$ as

$$
\tilde{T}(\partial)=\tilde{T}_{0}(\partial)^{2}-\tilde{T}_{1}(\partial)^{2}-\tilde{T}_{2}(\partial)^{2}
$$

with

$$
\begin{aligned}
& \tilde{T}_{0}(\partial):=\partial_{t}^{2}-c \Delta_{3}+d, \quad \tilde{T}_{1}(\partial):=e \Delta_{3}-f \\
& \tilde{T}_{2}(\partial):=g:=\sqrt{d^{2}-f^{2}}=\frac{2 \alpha}{J e} \sqrt{\frac{\alpha}{\rho}\left(\frac{\mu}{\rho}-\frac{\gamma+\varepsilon}{J}\right)} .
\end{aligned}
$$

We now set

$$
\tilde{T}_{\lambda}(\partial):=\tilde{T}_{0}+\lambda_{1} \tilde{T}_{1}+\lambda_{2} \tilde{T}_{2}=\partial_{t}^{2}+\left(\lambda_{1} e-c\right) \Delta_{3}+d-\lambda_{1} f+\lambda_{2} g
$$

for $\lambda_{1}^{2}+\lambda_{2}^{2} \leq 1$, and we observe that the family $\tilde{T}_{\lambda}(\partial)$ is uniquely quasihyperbolic in direction $N=(1,0,0,0)$. By Theorem 1 and the above remark we conclude that the operator $\tilde{T}(\partial)$ and thus also $T(\partial)$ is quasihyperbolic in $t$-direction. Since $T_{\mathrm{pr}}(N) \neq 0$, operator $T$ is also hyperbolic. Its unique fundamental solution with the properties (6) and (7) can be constructed from the fundamental solution of the iterated Klein-Gordon operator $T_{\lambda}(\partial)^{2}$ given in $[6$, p. 166]:

$$
E_{\tilde{T}_{\lambda}^{2}}=\frac{1}{8 \pi} J_{0}\left(\sqrt{\left(d-\lambda_{1} f+\lambda_{2} g\right)\left(t^{2}-\frac{|\mathbf{x}|^{2}}{c-\lambda_{1} e}\right)}\right) Y\left(\sqrt{c-\lambda_{1} e} t-|\mathbf{x}|\right)
$$

The result for $E_{T}$ is (cf. the computation in [7, p. 456 f.])

$$
E_{T}=\frac{1}{\rho J} E_{\tilde{T}}=\frac{1}{2 \pi \rho J} \int_{\lambda_{1}^{2}+\lambda_{2}^{2} \leq 1} E_{\tilde{T}_{\lambda}^{2}} \frac{\mathrm{d} \lambda_{1} \mathrm{~d} \lambda_{2}}{\sqrt{1-\lambda_{1}^{2}-\lambda_{2}^{2}}},
$$


and hence with $\lambda_{2}=\sqrt{1-\lambda_{1}^{2}} \cos \varphi$ and formula $6.684,1$ in [3]

$$
\begin{aligned}
E_{T}= & \frac{1}{16 \pi^{2} \rho J} \int_{-1}^{1} \int_{0}^{\pi} J_{0}\left(\sqrt{\left(d-\lambda_{1} f+g \sqrt{1-\lambda_{1}^{2}} \cos \varphi\right)\left(t^{2}-\frac{|\mathbf{x}|^{2}}{c-\lambda_{1} e}\right)}\right) \\
& \times Y\left(\sqrt{c-\lambda_{1} e} t-|\mathbf{x}|\right) \mathrm{d} \varphi \mathrm{d} \lambda_{1} \\
= & \frac{1}{16 \pi \rho J} \int_{-1}^{1} J_{0}\left(\sqrt{\frac{1}{2}\left(A+\sqrt{A^{2}-B^{2}}\right)}\right) \\
& \times J_{0}\left(\sqrt{\frac{1}{2}\left(A-\sqrt{A^{2}-B^{2}}\right)}\right) Y\left(\sqrt{c-\lambda_{1} e} t-|\mathbf{x}|\right) \mathrm{d} \lambda_{1},
\end{aligned}
$$

where

$$
A:=\left(d-\lambda_{1} f\right)\left(t^{2}-\frac{|\mathbf{x}|^{2}}{c-\lambda_{1} e}\right), \quad B:=g \sqrt{1-\lambda_{1}^{2}}\left(t^{2}-\frac{|\mathbf{x}|^{2}}{c-\lambda_{1} e}\right) .
$$

This result can be further simplified and we conclude the following theorem.

Theorem 2. The micropolar operator

$$
T(\partial)=\left(\rho \partial_{t}^{2}-(\mu+\alpha) \Delta_{3}\right)\left(J \partial_{t}^{2}-(\gamma+\varepsilon) \Delta_{3}\right)+4 \alpha \rho \partial_{t}^{2}-4 \alpha \mu \Delta_{3}
$$

is hyperbolic for $\rho, \mu+\alpha, J, \gamma+\varepsilon>0$. For $\frac{\mu+\alpha}{\rho} \neq \frac{\gamma+\varepsilon}{J}$ its unique fundamental solution $E_{T}$ with support in $t \geq 0$ is given by

$$
\begin{aligned}
E_{T}= & \frac{1}{16 \pi \rho J} \int_{-1}^{1} J_{0}\left(\sqrt{\frac{(1-\lambda)(d+f)}{2}\left(t^{2}-\frac{|\mathbf{x}|^{2}}{c-\lambda e}\right)}\right) \\
& \times J_{0}\left(\sqrt{\frac{(1+\lambda)(d-f)}{2}\left(t^{2}-\frac{|\mathbf{x}|^{2}}{c-\lambda e}\right)}\right) Y(\sqrt{c-\lambda e t}-|\mathbf{x}|) \mathrm{d} \lambda \\
= & \frac{1}{16 \pi \rho J} \begin{cases}0 & \text { if } t \leq \frac{|\mathbf{x}|}{\sqrt{c+e}} \\
\int_{-1}^{\frac{c t^{2}-|x|^{2}}{e t^{2}}} J_{0}\left(\sqrt{\Lambda_{1}}\right) J_{0}\left(\sqrt{\Lambda_{2}}\right) \mathrm{d} \lambda & \text { if } \frac{|\mathbf{x}|}{\sqrt{c+e}}<t \leq \frac{|\mathbf{x}|}{\sqrt{c-e}} \\
\int_{-1}^{1} J_{0}\left(\sqrt{\Lambda_{1}}\right) J_{0}\left(\sqrt{\Lambda_{2}}\right) \mathrm{d} \lambda & \text { if } t>\frac{|\mathbf{x}|}{\sqrt{c-e}},\end{cases}
\end{aligned}
$$

where

$$
\begin{aligned}
& c:=\frac{1}{2}\left(\frac{\mu+\alpha}{\rho}+\frac{\gamma+\varepsilon}{J}\right), d:=\frac{2 \alpha}{J}, e:=\frac{1}{2}\left|\frac{\mu+\alpha}{\rho}-\frac{\gamma+\varepsilon}{J}\right| \\
& f:=\frac{1}{e}\left(c d-\frac{2 \alpha \mu}{\rho J}\right)=\frac{\alpha}{e J}\left(\frac{\alpha-\mu}{\rho}+\frac{\gamma+\varepsilon}{J}\right)
\end{aligned}
$$


and

$$
\begin{aligned}
\Lambda_{1,2} & :=\frac{(1 \pm \lambda)(d \mp f)}{2}\left(t^{2}-\frac{|\mathbf{x}|^{2}}{c-\lambda e}\right) \\
& =\frac{(1 \pm \lambda) \alpha(\rho e \mp \rho c \pm \mu)}{\rho J e}\left(t^{2}-\frac{|\mathbf{x}|^{2}}{c-\lambda e}\right) .
\end{aligned}
$$

Proof. In the text above everything has been shown except that $T(\partial)$ is hyperbolic also if $\frac{\mu+\alpha}{\rho}=\frac{\gamma+\varepsilon}{J}$. But anyway the hyperbolicity of $T(\partial)$ follows from Theorem 12.4.6. in [4], p. 115, since the term $4 \alpha \rho \partial_{t}^{2}-4 \alpha \mu \Delta_{3}$ is weaker than $T_{\mathrm{pr}}$.

\section{References}

[1] E. Cosserat and Cosserat, F.: Théorie des Corps Déformables Paris: A. Hermann 1909.

[2] J. Gawinecki and Kacprzyk, P.: Asymptotic solutions of the equations to the microelasticity theory. Biuletyn WAT XLII (1993)(12), 49 - 59

[3] I.S. Gradshteyn and Ryzhik, I. M.: Table of Integrals, Series and Products. New York: Academic Press 1980.

[4] L. Hörmander: The Analysis of Linear Partial Differential Operators II. Grundlehren der mathematischen Wissenschaften, Vol. 257. Berlin: SpringerVerlag 1983.

[5] W. Nowacki: Theory of Asymmetric Elasticity. Warsaw: Polish Scientific Publishers 1981.

[6] N. Ortner: Regularisierte Faltung von Distributionen 2: Eine Tabelle von Fundamentallösungen. Z. Angew. Math. Physik (ZAMP) 31 (1980), 155 - 173

[7] N. Ortner and Wagner, P.: Some new fundamental solutions. Math. Meth. Appl. Sci. 12 (1990), $439-461$.

Received 03.12.2003 